\title{
SILENCE MISDIAGNOSED: MUTISM, AUTISM OR SUBMISSIVENESS? DIAGNOSTIC BIAS IN COLLECTIVISTIC CULTURE
}

\author{
Syeda Namrah Mahmood ${ }^{1 凶}$, Ayesha Jabeen'
}

\section{ABSTRACT}

Selective Mutism (SM) is a rare condition which is even rarer in collectivistic cultures. Firstly, SM is most often mistaken/misdiagnosed as autism spectrum disorder (ASD) and other related conditions due to lack of awareness of SM and the presence of mimicking characteristics of autistic behaviors in SM. Secondly, submissive behaviors in collectivistic societies further veil this rare condition by considering SM rather desirable than a problem. Especially in situations where developmental delay is a comorbid condition it became a great challenge to correctly diagnose among related conditions. We had a twelve years old girl with developmental delays who remained falsely diagnosed with ASD for five years. We have highlighted the diagnostic issues in SM and other related conditions especially ASD that might hinder the development of an appropriate management plan. The study also emphasized the need to develop a standardized assessment tool to diagnose SM considering cultural aspects into account.

KEY WORDS: Selective Mutism (MeSH); Autism Spectrum Disorder (MeSH); Submissiveness (Non-MeSH); Developmental delays (Non-MesH); Collectivistic culture (Non-MeSH).

THIS ARTICLE MAY BE CITED AS: Mahmood SN, Jabeen A. Silence Misdiagnosed: Mutism, Autism or Submissiveness? Diagnostic Bias in Collectivistic Culture. Khyber Med Univ J 2018;10(3):159-62.

\section{INTRODUCTION}

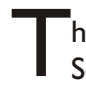

he historical origin of the concept of

Selective Mutism (SM) is dated back to $19^{\text {th }}$ century where it was first named as aphasia voluntaria describing a disorder in which individual appears to avoid speaking voluntarily in specific situations.' However, in latest versions of Diagnostic and Statistical Manual (DSM) V (DSM V) the term elective was finally modified to selective reflecting it as a childhood condition specified by a consistence failure to speak in selective social situations where the speech is expected. ${ }^{2}$ Children having SM appear to exhibit normal communication at home with parents and siblings, however, consistence failure to speak occurs mostly at school setting or any other less familiar social setting. ${ }^{3}$ SM exhibits a multidimensional symptom presentation; it may have a variety of comorbidities with other disorders including speech and language pathologies, elimination disorders, neurotic problems, intellectual disability and autism spectrum disorder (ASD). ${ }^{4}$

The analysis of available literature suggests that SM is most often inaccurately misdiagnosed with ASD due to mimicking of certain behavior characteristics present in both SM and ASD children including shyness, lack of social interaction and verbal speech, being fearful and often oppositional etc. ${ }^{5}$ However, the characteristics of ASD which markedly distinguishes it from SM or other developmental disorders include; neurodevelopmental in nature unlike SM, absence of social reciprocity and presence of restricted, stereotypic and repetitive variety of interests, behaviors and activities. ${ }^{6}$

Furthermore, the role of culture also plays a crucial role in the fate diagnosis. It is a well-established fact that cultural norms provide the perspective which defines either a childhood behavior is problematic or not. ${ }^{7}$ In a collectivistic culture like ours, shyness or submissiveness is not only considered desirable but
I Institute of Clinical Psychology, University of Management and Technology, Lahore, Pakistan

Email ${ }^{凶}$ : namrahbukhari@gmail.com

Contact \#: +92-3334144474

Date Submitted: March 18, 2018

Date Revised: July 17, 2018

Date Accepted: August 16, 2018

also determines adults' attitude with a child which promotes limited participation and shyness among children in such societies. ${ }^{8}$ Therefore, it would not be wrong to say that an already rare condition (SM) is even rarer in developing world because: first most of the professionals are unaware about the condition, second they prefer more glamorized disorders like ASD over SM and last, they simply do not consider it a problem as it contains characteristics which are more desirable in collectivistic culture.

More importantly, the diagnosis became more challenging and confusing as SM most commonly co-occur with developmental delays, for example, Kristensen ${ }^{9}$ founded that children with SM showed comorbidity with developmental delays (DD) up to $68.5 \%$. DD already affects child's speech development suggesting less use of verbal communication which is further exacerbated by SM. ${ }^{10}$ Furthermore, another notion is becoming popular that SM may serve as a protective factor helping children conceal their developmental disabilities under their silence.' This is the irony of fate that lack of standardized assessment of SM especially in the face of DD and paucity of empirical research along with lack of general awareness of SM create crucial hurdles in assisting children with SM and comorbid conditions. The data suggests that such children misdiagnosed as autistic, language delayed etc. saddle them with inappropriate or ineffective interventions leading towards farreaching psychological consequences." The aim of the current study is to address one of the most ignored developmental conditions, SM, by diverting attention of the professionals towards current diagnostic issues of SM (from differential diagnosis to cultural factors) and to develop a culture specific standardized assessment to make an accurate diagnosis by eliminating existing confusions. 


\section{CASE PRESENTATION}

The patient was II years old girl who belonged to a low socio-economic status and lived in a joint family system. The client was admitted to the special education school of city Lahore approximately six years before. At the time of recent referral of patient to therapist she was presented with the complaints of being shy and nonparticipative in school activities, avoidance of interaction with other school mates, unable to establish and maintain eye contact, lack of social reciprocity in terms of non-verbal communication and total absence of verbal communication. The detailed evaluation revealed strong genetic and postnatal history contributing in the disability of patient. The consanguineous marriages had been in practice for generations in patient's family which resulted in a number of cases of disabilities among children of her family. The postnatal history of patient elucidated that patient suffered from high grade fever within a month of her birth and she also suffered from pneumonia after one month of her birth. During infancy her mother was not being able to breastfeed patient as patient faced difficulty in sucking.

The caregiver reported that patient's developmental milestones were delayed as well, for example, patient achieved developmental tasks of neck holding, walking, sample language ( 2 to 3 words) and bladder control at the approximate ages of 2.5 years, 4 years, 10 years and 7 years, respectively. The problems of incontinence, poor self-help and social skills and temper tantrums persisted till the time she started schooling. However, all her presenting problems gradually faded away within 2 to 3 years except for her social skills which mainly included poor eye contact and lack of social reciprocity in terms of both verbal and non-verbal communication. The patient at the time of admission at school was perceived as deaf-mute and hence her assessment and management ignored the area of social skills. However, after few years she was suspected of being capable of speaking but keeping her social skills into consideration she was again misdiagnosed with ASD. The teacher of the patient stated that although patient was an aloof child but it never bother her teachers as she was most submissive among all students of her class and never gave tough time to teachers. More importantly, patient was never witnessed to communicate (both verbally and non-verbally) in school with anyone except for her cousin with whom she found twice or thrice while secretly talking. However, one of her family members reported that patient did exhibit speech and communicate frequently using simple sentences (4-5 words) at home. Moreover, patient continued to take speech therapy sessions since she was admitted to the school but no improvement was reported by her speech therapist in patient's speech and her social reciprocity.

A multimodal and comprehensive approach had been adopted in order to assess patient's presenting problems including; behavioral observations, interviews of family members and professionals (teachers, audiologist, psychiatrist and speech pathologist) and administration of standardized measures.

The direct and indirect observation of patient by the therapist in a number of settings (from classroom to playground) revealed that patient had no hearing impairment; she exhibited three to four words speech with adequate articulation but marked avoidance of verbal communication and participation in social activities at school but not at home was observed and reported.

The formal evaluation of patient's presenting problems involved the administration of Childhood Autism Rating Scale (CARS), ${ }^{12}$ Portage Guide to Early Education (PGEE) ${ }^{13}$ and Bender Gestalt Test (BGT). ${ }^{14}$ The administration of CARS involved the rating of both parents and teachers separately which showed a marked discrepancy between both. According to the ratings by parents patient fell in the category of minimal to no symptoms of ASD (16.5) whereas the ratings by teacher suggested the presence of mild to moderate symptoms of ASD (3I). The potential differences of ratings by parents and teachers lied on items; relating to people, imitation and verbal and non-verbal communication with a difference of $I .5$ points, 2 points, 2 points and $\mathrm{I} .5$ points, respectively.

PGEE was administered to assess patient's functioning on five developmental areas. ${ }^{13}$ The profile of patient showed that except for cognitive (3-4 years) and language areas ( $\mathrm{I}-2$ years) other areas of PGEE are developed till 56 years of age. The analysis of patient's profile revealed that her profile was unusual and inconsistent, for example, a significant discrepancy was found between socialization area and other two areas closely related to socialization i.e. cognitive and language. The qualitative analysis revealed that this significant difference was not due to patient's low cognitive skills rather due to more verbal skills required by most of the tasks in cognitive area than socialization area of PGEE.

The scores of patient in cognitive area of PGEE suggested to assessing her cognitive abilities using some non-verbal measure. Therefore, BGT was preferred to assess the developmental maturation of patient through non-verbal means, using a unique system developed by Koppitz for children. ${ }^{14,15}$ The BGT profile reflected that developmental maturation of patient fell between 5-7 years or even above in some designs suggesting a significant discrepancy with the outcome of cognitive area of PGEE. This discrepancy of 3 years or above in outcomes of both measures provided a strong explanation of unusual profile of patient on PGEE. It could be assumed that patient performed low on cognitive area of PGEE as it required verbal skills whereas BGT did not require any verbal ability she scored better in developmental maturation. Therefore, it provided substantial evidence that lack of generalizability of speech had affected patient's performance in cognitive area rather than developmental delay in cognitive area had been affecting her language abilities. Therefore, it could be assumed that poor performance on language area was not due to the absence of speech or social reciprocity but due to her social anxiety or SM. Moreover, the developmental maturation of patient as revealed by BGT was also a reflection of patient's well developed perceptual and motor abilities ( 7 years or above) as reproduction of figures require coordination of both skills. 


\section{DISCUSSION}

This case study presented various assessment and diagnostic challenges due to the multidimensional nature of SM, as reported in literature. The issues which led towards misdiagnosis of patient as highlighted by current study include; resembling features of ASD and SM, acceptance of SM characteristics (such as shyness, restricted participation) in collectivistic culture and comorbidity of DD with SM. Before discussing these aspects in the light of previous literature in detail, it is important to see how patient's case was conceptualized and evidence was linked with SM.

The patient's case was conceptualized in light of bio-psychosocial model which suggests genetic predisposition as well as consanguinity as predisposing biological factors. Both are well-known risk factor for genetic disorders, including diseases and syndromes that present with intellectual and developmental disabilities. $^{16,17}$ Furthermore, previous literature suggests that development of 'triadic' (person-person-world) social interactions may be influenced by limited information-processing capacities in infants with developmental delays, through a complex socially-mediated developmental trajectory. ${ }^{18}$ So, it could be assumed that this limited information processing capacities might have initially served as a precipitating factor in the development of SM in patient. However, later on patient might use $\mathrm{SM}$ as a protecting factor in concealing her developmental problems or weaknesses.' Moreover, other social factors that seemed to influence her anxiety and speech problem include lack of encouragement by teachers to communicate and absence of proper management previously. Furthermore, frequent interaction of patient with her cousin (who had developmental disability) also exacerbated her symptoms as patient might not feel need to interact with strangers which seemed threatening to her. This way rather than I

earning from other children as well, she only tried to model her cousin who was having speech problems too.

The literature has suggested that globally an over diagnosis of ASD has been observed; one of the reasons behind this is the unfamiliarity or lack of awareness of professionals with features of rare conditions like SM. ${ }^{4,5}$ The second aspect responsible for SM misdiagnosis highlighted by this study was that characteristics of SM including shyness or lack of interaction might not consider as problematic in collectivistic cultures rather such behaviors are encouraged, therefore, SM is often overlooked. ${ }^{8}$ The last factor was the presence of DD in patient which posed challenges in making a diagnosis and formulating intervention plan. Literature has suggested that DD undermines the presence of other comorbid conditions leading to inefficient diagnosis. 10,19

Moreover, currently the diagnosis of SM relied on indirect assessment measures including behavioral observation, functional assessment, interviews with parents and teachers and adherence to diagnostic criteria. The unavailability of standardized assessment often mislead to false diagnosis especially in the presence of DD.6, 19 The case study highlights the need of a culturally reliable scale to not only to correctly assess SM in a shorter time span but to help devising an effective intervention plan.

\section{REFERENCES}

I. Sharkey L, McNicholas F. More than 100 years of silence, elective mutism. Eur Child Adolesc Psychiatry 2008; I 7(5):255-63. DOI: 10.1007/s00787-007-0658-4.

2. American Psychiatric Association. Diagnostic and statistical manual of mental disorders. $5^{\text {th }}$ ed. Washington, DC: American Psychiatric Publishing; 2013.

3. Sharp WG, Sherman C, Gross AM. Selective mutism and anxiety: A review of the current conceptualization of the disorder. J Anxiety Disord 2007;2I(4):568-79. DOI: 10.1016/j.janxdis.2006.07.002.

4. Wong P.Selective mutism: a review of etiology, comorbidities, and treatment. Psychiatry (Edgmont) 2010;7(3):23-31

5. Kehle TJ, Madaus MR, Baratta VS, Bray MA. Augmented self-modeling as a treatment for children with selective mutism. J Sch Psychol 1998;36(3):247-60. DOI:

\section{I0.1016/S0022-4405(98)000 I3-2.}

6. Ahmad S, Mahmood Z. Differential diagnosis of Autism and Mental Retardation: issues and possible solutions. FWU J Soc Sci 201 I;5(I):16-33.

7. Gudiño OG, Lau AS. Parental cultural orientation, shyness, and anxiety in Hispanic children: An exploratory study. J Appl Dev Psychol 20I0;3।(3):202-I0. DOI: 10.1016/j.appdev.2009.12.003.

8. Chen X, DeSouza AT, Chen H, Wang L. Reticent behavior and experiences in peer interactions in Chinese and Canadian children. Dev Psychol $2006 ; 42(4): 656$. DOI: 10.1037/00I2-1649.42.4.656.

9. Kristensen H. Selective mutism and comorbidity with developmental disorder/delay, anxiety disorder, and elimination disorder. J Am Acad Child Adolesc Psychiatry 2000;39(2):24956. DOI: 10.1097/00004583200002000-00026.

10. Alpaslan AH, Koçak U, Coşkun KŞ, Çobanoğlu C. A case of severe longterm selective mutism in a child with intellectual disability. Int J Dev Disabil 2016;62(2):131-5. DOI: 10.1 179/ 20473877 I 5 Y.0000000003.

II. Shipon-Blum E. When the words just won't come out: Understanding selective mutism. The Selective Mutism Group Childhood Anxiety Network (SMG-CAN) 2007. [Cited on: March 10, 2018]. Accessed from URL: http://www.arnoldpalmer hospital.com/ /media/files/practice s/neuropsychology-consultantsaph/selective-mutism2.pdf.

12. Schopler E, Reichler RJ, Renner BR. The childhood autism rating scale (CARS). Los Angeles, CA: Western Psychological Services; 2002.

13. Bluma SM, Searer MS, Frohman AH, Hilliard JM. Portage guide to early education- revised edition. Portage, Wisconsin: Cooperative Educational Service Agency; 1976.

14. Koppitz EM. The Bender gestalt test for young children. Harcourt Health Sciences Group 1963.

15. Koppitz EM. The Bender gestalt test for young children: research and 
applications. New York, NY: Grune \& Stratton; 1975.

16. Stigler SM. Darwin, Galton and the statistical enlightenment. J R Stat Soc Series A (Statistics in Society). $2010 ; 173(3): 469-82$. DOI: $10.1111 /$ j. $1467-985 \times .2010 .00643 . x$.
17. Colvin L, Jurenka SB, Van Allen MI. Down syndrome. In: Hisami FM, Weissman SM, Martin G, editors., editors. Chromosomal instability and aging. New York Marcel Dekker; (2003). p. 44I-63.

18. Moore DG, Oates JM, Hobson RP,
Goodwin J. Cognitive and social factors in the development of infants with Down syndrome. Downs Syndr Res Pract 2002;8(2):43-52.

19. Girimaji SC. Clinical practice guidelines for the diagnosis and management of children with

\section{AUTHORS' CONTRIBUTIONS}

Following authors have made substantial contributions to the manuscript as under:

SNM: Concept \& study design, acquisition, analysis and interpretation of data, drafting the manuscript, critical review, final approval of the version to be published.

AJ: Acquisition of data, critical review, final approval of the version to be published.

Authors agree to be accountable for all aspects of the work in ensuring that questions related to the accuracy or integrity of any part of the work are appropriately investigated and resolved.

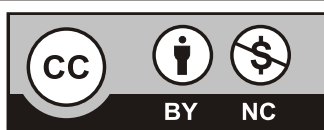

BY NC

\section{CONFLICT OF INTEREST}

Authors declared no conflict of interest GRANT SUPPORT AND FINANCIAL DISCLOSURE NIL
This is an Open Access article distributed under the terms of the Creative Commons Attribution-NonCommercial 2.0 Generic License.
KMUJ web address: www.kmuj.kmu.edu.pk

Email address: kmuj@kmu.edu.pk 